\title{
Interférences
}

Ars scribendi

$5 \mid 2009$

Historia / Persona

\section{La trajectoire de Lucius Domitius Ahenobarbus chez Lucain}

Fabrice Galtier

\section{OpenEdition}

1 Journals

Édition électronique

URL : http://journals.openedition.org/interferences/901

DOI : 10.4000/interferences.901

ISSN : $1777-5485$

Éditeur

HiSoMA - Histoire et sources des Mondes antiques

Édition imprimée

Date de publication : 1 janvier 2009

ISSN : 1777-5485

Référence électronique

Fabrice Galtier, «La trajectoire de Lucius Domitius Ahenobarbus chez Lucain », Interférences [En ligne], 5 | 2009, mis en ligne le 10 juillet 2014, consulté le 15 septembre 2020. URL : http://

journals.openedition.org/interferences/901; DOI : https://doi.org/10.4000/interferences.901

Ce document a été généré automatiquement le 15 septembre 2020.

Tous droits réservés 


\title{
La trajectoire de Lucius Domitius Ahenobarbus chez Lucain
}

\author{
Fabrice Galtier
}

1 Dans le volume des Entretiens de la Fondation Hardt consacré à Lucain, B. Marti, intervenant à la suite de l'exposé de $\mathrm{P}$. Grimal sur le poète et l'Histoire ${ }^{1}$, cite à deux reprises le cas de Lucius Domitius Ahenobarbus pour illustrer le phénomène de l'inexactitude historique dans la Pharsale ${ }^{2}$. La manière dont ces deux mentions sont formulées permet de préciser la problématique soulevée par le traitement du personnage dans l'épopée lucanienne. En effet, B. Marti évoque d'abord l'action de Domitius à Corfinium, qui, selon elle, n'est pas rapportée exactement ; immédiatement après, selon un raisonnement implicite qui pose le premier constat comme une prémisse, elle indique que Lucain «fait un héros de Domitius ». Ce qui apparaît donc, c'est que le compte rendu des actes du chef pompéien, considéré comme déviant par rapport à la réalité historique, détermine une conception du personnage tout aussi éloignée de la véritable nature de l'individu. Mais affirmer un tel constat, c'est partir du principe que serait parfaitement établie cette vérité historique dont Lucain s'écarterait. Or, rien n'est moins sûr. C'est pourquoi analyser la manière dont le poète représente le comportement de Domitius implique de s'interroger sur la validité de son traitement historiographique. Cette étape préliminaire permet d'éclairer la caractérisation du personnage en fonction de la double confrontation dialectique qui le met aux prises avec César.

2 Il faut donc considérer quelle image nous est offerte de l'action accomplie par Domitius dans les trois épisodes de la guerre civile où la plupart des auteurs anciens s'accordent à lui voir jouer un rôle important: la prise de Corfinium, qui demande un examen particulièrement détaillé, la chute de Marseille et la bataille de Pharsale.

3 Donnons au préalable sur l'individu en question les rares indications qui semblent ne faire aucun doute. Lucius Domitius Ahenobarbus est le trisaïeul de Néron. Il s'agit là d'un point sur lequel nous serons bien évidemment obligé de revenir. Consul en 54, il est nommé proconsul des Gaules en remplacement de César, dont il est l'un des ennemis les plus acharnés ${ }^{3}$. Au moment où le futur dictateur conquiert le Picenum, en 
février 49, Domitius se trouve à Corfinium. Il tente de faire détruire le pont qui franchit le cours d'eau barrant l'accès à la ville, mais l'avant-garde césarienne chasse ses hommes. Assiégé et privé du soutien militaire de Pompée, il est finalement livré à César qui l'épargne et le renvoie, libre. Par la suite, il soutient les Marseillais lors du siège de leur cité et parvient à s'en échapper juste avant qu'elle ne capitule. Enfin, il participe à la bataille de Pharsale à l'issue de laquelle il est tué en 48.

4 L'épisode de Corfinium possède un caractère symbolique que Lucain ne peut méconnaître. Il constitue une étape essentielle dans la progression de César vers l'Italie centrale et un revers d'importance pour Pompée, qui se trouve privé des légions dont disposait Domitius ${ }^{4}$. De plus, il est le premier théâtre d'affrontement entre César et celui qu'on avait nommé pour lui succéder comme proconsul des Gaules et qui se présentait depuis longtemps comme l'un de ses ennemis politiques les plus acharnés. Enfin, il est marqué par un fait qui va frapper les esprits : le pardon accordé par César à Domitius, après que celui-ci lui eut été livré. Ce geste permet au futur dictateur de faire étalage de ce qu'on appellera sa clémence ${ }^{5}$. L'épisode concentrait donc de tels enjeux d'ordre politique et idéologique qu'il ne pouvait échapper aux manipulations. C'est pourquoi il faut demeurer extrêmement prudent quand on accuse Lucain de déformer une réalité historique que les différentes versions de l'événement ont, de toute manière, considérablement brouillée.

Lucain, d'ailleurs, élude d'emblée une question qui agite encore aujourd'hui les spécialistes de la période : celle des responsabilités exactes de Domitius et de Pompée dans la défaite. Il disjoint donc les faits et traite de manière indépendante l'épisode de Corfinium et les mouvements de Pompée ${ }^{6}$. Avant Corfinium, la description des Apennins et de la retraite des autres chefs crée une rupture. Après le récit, le poète revient à Pompée mais fait en sorte que ses actes soient sans lien apparent avec ce qui vient d'arriver à Domitius. Usant d'un effet de trompe-l'œil, Lucain souligne que Pompée ignore la capture du proconsul lorsqu'il tente, en vain, de galvaniser ses hommes ${ }^{7}$. Mais, s'il s'est nécessairement trouvé un laps de temps durant lequel Pompée n'a pas eu connaissance du sort de Domitius, cela ne signifie aucunement, comme le récit le laisse croire, qu'il ignorait tout de la tentative de Domitius pour barrer la route à César. Il avait désapprouvé cette décision et tenté d'obtenir qu'il le rejoignît avec ses trente cohortes, afin de renforcer le corps de troupes qu'il était en train de constituer. De son côté, Domitius lui avait réclamé des renforts, conscient que, s'il demeurait seul, il ne pourrait résister longtemps. Il semble qu'il ait été fermement décidé à infliger sa première défaite à César, quitte à forcer la main à Pompée. Mais ce dernier refusa de céder à sa demande. Domitius fut pris, et ses cohortes se rallièrent à l'ennemi. En jouant sur un procédé d'occultation, Lucain présente comme indépendantes deux lignes de conduite qui, en réalité, eurent des implications réciproques. Il évite ainsi de poser la question de la responsabilité de Pompée dans la prise de Corfinium ${ }^{8}$, et surtout, il estompe la faute de Domitius dont l'entêtement a peut-être été lié à une incapacité foncière à juger sainement la situation ${ }^{9}$. Il faut ajouter qu'un tel parti pris permet d'empêcher d'établir un quelconque lien de cause à effet entre la chute de la cité et le départ de Pompée de Lucérie pour Brindes. Or c'est justement ce lien que crée César au début du chapitre 24 : «Pompée, écrit-il, une fois connus les événements qui s'étaient déroulés à Corfinium, quitte Lucérie pour Canusium et ensuite pour Brindes ${ }^{10}$ ». En réalité, Pompée quitte Lucérie deux ou trois jours avant une défaite qu'il avait probablement prévue ${ }^{11}$. 
6 En dehors des questions d'ordre stratégique, le comportement de Domitius lors du siège et de la prise de la ville a fait l'objet de versions discordantes, voire profondément contradictoires. Les divergences se concentrent sur deux points essentiels.

7 Tout d'abord Domitius a-t-il voulu trahir ses propres troupes? Dans les chapitres 16 à 23 du livre I de son Bellum Ciuile, César fait un compte rendu extrêmement précis de ce qui s'est déroulé dans Corfinium à partir du moment où le proconsul s'y est retrouvé assiégé. Selon lui, après avoir reçu la lettre où Pompée lui refusait les renforts, il ment à son état-major, annonçant que Pompée va rapidement se porter à leur secours. Dans le même temps, il s'entretient avec ses proches et leur révèle qu'il est résolu à prendre la fuite. Mais, écrit César, «comme la physionomie de Domitius ne s'accordait pas avec son langage, que tous ses actes marquaient moins de confiance et de fermeté qu'il n'en avait montré les jours précédents, qu'il s'entretenait souvent avec ses familiers en de mystérieux conciliabules, contrairement à ses habitudes, qu'il évitait les rassemblements et les réunions, la vérité ne put pas être cachée et tenue secrète plus longtemps ${ }^{12}$ ». César prend soin d'indiquer qu'une partie des officiers se joignent aux hommes de troupe pour discuter de la situation. Indignés de l'attitude de leur chef et décidés à sauver leur peau, ils le font prisonnier et entrent en pourparlers avec le vainqueur des Gaules à qui ils livrent à la fois leur état-major et la ville. Dans la version de Lucain, le seul acte de trahison évoqué est celui des hommes de Domitius qui, " portes ouvertes, entraînèrent leur chef prisonnier et l'arrêtèrent aux pieds de son arrogant concitoyen ${ }^{13}$.

Dans sa correspondance, Cicéron ne fait aucune mention d'une quelconque désertion de Domitius. Ainsi, dans la lettre qu'il écrit à Atticus le 23 ou 24 février, il mentionne la rumeur selon laquelle le proconsul se serait rendu avec les siens, une fois connue la décision de Pompée :

Domitium autem aiunt re audita et eos qui una essent se tradidisse ${ }^{14}$.

Le caractère extrêmement vague de l'information donnée par Cicéron, que renforce le terme aiunt, doit autant nous inciter à la prudence que la troublante précision du compte rendu césarien. Dans celui-ci, la longue description de l'attitude suspecte de Domitius est rendue à travers deux séries de notations: la première porte sur le caractère contradictoire de son comportement entre son langage et son visage, entre sa fermeté passée et la fébrilité présente, la deuxième sur le changement de nature de ses réunions. Il fuit les rassemblements, mais multiplie les entretiens en petit comité. C'est ainsi que la vérité aurait éclaté. Mais, si l'on s'en tient à ce récit, l'armée ne pouvait nourrir, au mieux, que de vagues soupçons. L'expression diuulgato Domiti consilio ${ }^{15}$, qui ouvre le chapitre 20 , ne renvoie, en réalité, à aucune révélation concrète. Les soldats semblent deviner le projet de Domitius, qui apparaît par la suite comme une évidence, sans que rien ne vienne le confirmer. Ce qui rend d'autant plus suspecte cette version des événements est le fait qu'elle permet d'envoyer Domitius rejoindre la cohorte de pompéiens apeurés dont les actions indignes jalonnent le Bellum Ciuile ${ }^{16}$. Or justement, le volontarisme dont il fait preuve, au début du siège, contraste avec l'attitude des autres généraux, fuyant devant la progression foudroyante de l'ennemi dans le Picenum. Suétone, lorsqu'il évoque la généalogie de Néron, entend montrer que les vices de chacun de ses ancêtres se retrouvèrent en lui ${ }^{17}$. Dans les lignes qu'il consacre au trisaïeul du prince, il était tentant d'insérer un exemple de couardise qui aurait préfiguré la lâcheté bien connue du dernier des Julio-Claudiens. Le biographe mentionne bien un tel acte de la part de Domitius, mais pas le projet de fuite évoqué 
par le compte rendu césarien ${ }^{18}$. Constatons enfin que le proconsul conserva par la suite la confiance de Pompée, puisqu'on lui confia, entre autres, le commandement d'une partie des troupes, à Pharsale ${ }^{19}$. En admettant qu'il ait bien été livré par ses soldats, c'est fort probablement pour des motifs autres que ceux mentionnés par César. Dans cet ordre d'idées, diverses hypothèses se présentent. Selon K. von Fritz, il est possible que Domitius ait décidé de quitter en secret la ville pour parler personnellement à Pompée et tenter de le convaincre. Pour A. Burns, la tentative de fuite, si elle a eu lieu, serait la conséquence de la mutinerie et non sa cause ${ }^{20}$. Mais les deux exégètes soulignent aussi la crédibilité de la version présentée par Dion Cassius : Domitius se serait finalement résolu à tenter un passage en force pour rejoindre Pompée. C'est ce projet, et non une désertion, qui aurait provoqué la mutinerie de ses troupes, craignant que ce départ fût tenu pour une fuite ${ }^{21}$. Les hommes ont donc pu se sentir trahis par une décision de leur chef qui ne correspondait plus au plan initial consistant à tenir la ville jusqu'à l'arrivée des renforts. De fait, la version de Dion Cassius est d'autant plus intéressante qu'elle permettrait d'éclairer la manipulation césarienne. Celle-ci aurait consisté à transformer en fuite individuelle un projet d'évacuation que la troupe a pu considérer comme un lâche abandon ou une entreprise vouée à l'échec ${ }^{22}$. Il est tentant de penser, comme A. Burns, que le récit de Dion Cassius reprendrait, de première ou de seconde main, la version livienne ${ }^{23}$. Mais, plus généralement, nous souscrirons volontiers à l'hypothèse émise par G. Zecchini concernant l'existence d'une tradition pro-républicaine dans laquelle Lucain aurait puisé, tradition illustrée notamment par Sénèque le Père ${ }^{24}$.

Passons au deuxième point: le suicide raté de Domitius. Plusieurs auteurs en font mention, mais avec des variantes. Le récit le plus célèbre est celui qu'en offre Sénèque, dans le De beneficiis. Domitius, bloqué dans Corfinium, demande à son esclave-médecin de lui donner du poison afin de mourir les armes à la main ${ }^{25}$. Le serviteur lui procure un breuvage qu'il sait inoffensif. C'est ainsi qu'il sauve son maître, César ayant entretemps annoncé qu'il épargnerait les défenseurs de la ville. C'est manifestement le même épisode qu'évoque Suétone, bien qu'il n'en mentionne pas les circonstances exactes. Mais, cette fois, il s'agit de montrer un manque de fermeté face à la mort qui préfigure les derniers instants de Néron. C'est dans un moment de désespoir qu'il avale le poison, et il le vomit sous l'effet de la peur. Cependant, le double jeu du médecin est conservé ${ }^{26}$. Chez Plutarque également, l'esclave donne un ersatz de poison à son maître, et c'est un Domitius soulagé et heureux d'être en vie qui va témoigner toute sa reconnaissance à un César dont l'étonnante philanthropia est présentée comme exemplaire ${ }^{27}$. On ne trouve rien de tel dans le texte du Bellum Ciuile, mais il y est écrit que le pompéien Lentulus Spinther, entré en pourparlers avec César pour lui demander de l'épargner, avoue que «certains sont si terrorisés qu'ils sont amenés à prendre des résolutions funestes pour leur vie ${ }^{28}$. Il pourrait s'agir là d'une référence implicite à Domitius, dont le projet de suicide, à peine évoqué, serait en outre dévalorisé par son motif : la terreur qu'il aurait éprouvée à l'idée d'être livré à l'ennemi. Si tel est le cas, il y aurait bien eu une volonté de la part du Pompéien de mettre fin à ses jours, ce qui aurait donné naissance à une double tradition faisant de lui soit un Caton avant l'heure, soit un couard. Cependant, et contrairement à ce qu'ont affirmé plusieurs commentateurs, peut-être marqués par l'épisode du De beneficiis, cette tentative de suicide n'est jamais relatée, en tant que telle, par Lucain, qui passe directement des préparatifs du siège au face à face entre le captif et César. En revanche, mais nous y 
reviendrons, le premier réclame au second d'être exécuté, ce que refuse celui-ci, préférant faire éclater sa lenitas en lui accordant la vie sauve et la liberté.

Il n'y a pas de raison de douter que, par la suite, Domitius ait bel et bien aidé les Marseillais assiégés par l'armée césarienne, même si on peut soupçonner le vainqueur des Gaules d'avoir, une fois de plus, présenté les faits de manière à noircir ses adversaires ${ }^{29}$. Il semble ainsi à peu près certain que le proconsul parvint à s'enfuir juste avant que la ville ne capitule. Mais de son action aux côtés des Marseillais, de la manœuvre qui permit à son navire de franchir les lignes ennemies, Lucain ne dit mot. Dans la version qu'il présente du siège de Marseille, Domitius n'est jamais mentionné ${ }^{30}$. Pourtant, sa participation à cet épisode est suggérée plus loin, lors de sa mort à la bataille de Pharsale. Lucain écrit en effet à son propos :

quem clades fata per omnis / ducebant : nusquam Magni fortuna sine illo / succubuit ${ }^{31}$.

Ainsi, le pompéien meurt totiens uictus a Caesare ${ }^{32}$. Il est clair que ces expressions ne renvoient pas à une seule défaite antérieure - Corfinium - mais à deux, la deuxième étant la chute de Marseille, avant l'ultime désastre que constitue Pharsale ${ }^{33}$.

Poursuivant son portrait de Lucius Domitius en lâche, César accorde à ce dernier une mort proche de l'indignité :

L. Domitius ex castris in montem refugiens, cum uires eum lassitudine deficissent, ab equitibus est interfectus ${ }^{34}$.

En fait, Domitius devait accompagner le mouvement de retraite des débris de l'armée sénatoriale vers une colline située non loin du camp de Pompée ${ }^{35}$. Mais en disjoignant son cas de celui des troupes vaincues, César laisse croire qu'il s'est enfui seul. Il renforce l'impression de lâcheté par le contraste qu'il établit avec la mort héroïque du centurion Crastinus, frappé d'un coup d'épée en plein visage, donc face à l'ennemi ${ }^{36}$. Lucain, quant à lui, choisit délibérément de s'écarter de la vérité du détail historique en imaginant un ultime face à face symbolique et profondément épique entre César et Domitius, en plein champ de bataille ${ }^{37}$.

De cette rapide confrontation entre le récit lucanien et les autres textes portant sur les faits et gestes de Lucius Domitius Ahenobarbus, on peut d'ores et déjà tirer quelques leçons provisoires.

Tout d'abord, s'il apparaît nettement que Lucain prend des libertés avec la réalité historique, il ne la défigure pas. Le phénomène doit être d'autant plus relativisé si l'on prend en compte le genre dans lequel il s'exprime, la conception très large de la vérité historique que se faisaient les Anciens et le peu de certitudes que l'on a sur le détail des événements ${ }^{38}$. Il ne nous semble pas, en particulier, que le poète métamorphose en héros un être qui, à l'origine, aurait été totalement veule. La lâcheté supposée de Domitius est largement liée à ce qu'en rapporte César. Ce dernier a manifestement cherché à dénigrer un homme qui pouvait apparaitre comme un symbole de la défense des institutions. De fait, une tradition pro-républicaine favorable au personnage et opposée à la propagande césarienne a dû exister, sur laquelle le poète s'est appuyé ${ }^{39}$. On s'aperçoit donc que, pour comprendre le travail de Lucain sur Domitius, il était essentiel d'examiner le portrait à charge qu'en faisait César. Incontestablement, Lucain, pour reprendre une expression de P.M. Martin, « réplique point par point à l'auteur du Bellum Ciuile ${ }^{40} »$. Nous voyons ici l'illustration parfaite de ce que M. Rambaud avait souligné dans un article célèbre sur ce sujet ${ }^{41}$. Enfin, on notera que, pour façonner l'image de son personnage, le poète recourt à deux procédés complémentaires: le premier, mis en valeur par J. Masters, consiste à combler les «blancs» de l'Histoire : 
«filling in the gaps ${ }^{42}$ ». Ainsi Lucain imagine les pensées de Domitius lorsque César lui accorde la vie sauve et, de façon plus hardie, invente une rencontre entre les deux hommes sur le champ de bataille. Il a pu être constaté que le poète recourt aussi au procédé inverse : il ménage de nombreux blancs dans le récit, qui lui permettent de redessiner la trajectoire de Domitius selon un axe idéologiquement déterminé.

Nous l'avons dit, chez César, Domitius est un être en fuite perpétuelle, cherchant à éviter les conséquences des désastres où il se trouve impliqué : il veut fuir Corfinium quand le piège se referme sur lui, il fuit Massilia sur le point de tomber, il fuit le camp de Pompée après la défaite de Pharsale ${ }^{43}$. Chez Lucain au contraire, Domitius reçoit l'épithète de pugnax, et ce, dans chacun des épisodes où il apparait : dans tous les cas, il est qualifié ainsi lorsque son nom est cité pour la première fois ${ }^{44}$. Cette marque d'opiniâtreté le définit d'emblée à chacune de ses entrées en scène, suggérant qu'entre Corfinium et Pharsale, rien n'a pu altérer sa nature profonde. Nous n'insisterons pas sur les marques les plus évidentes d'une fermeté d'âme qui s'affirme plus particulièrement devant la mort. Lorsqu'on l'amène, ligoté, aux pieds de César, c'est uultu minaci et recta ceruice qu'il réclame le fer ${ }^{45}$, semblant ainsi provoquer son ennemi pour être sûr de ne pas échapper au supplice. Dans le chant VII, l'héroïsation de Domitius joue sur une réalité, non pas historique, mais historiographique. Lucain écrit en effet :

Mors tamen eminuit clarorum in strage uirorum / pugnacis Domiti ${ }^{46}$.

Or, il est tout à fait exact qu'il est le seul Pompéien connu dont on ait rapporté la mort durant la bataille de Pharsale. Se saisissant de cette aubaine offerte par l'historiographie, Lucain en profite pour créer une mors illustris uiri. Domitius s'effondre donc en plein combat, succombant à ses blessures, parmi les partisans de Pompée ${ }^{47}$. L'ultime dialogue avec César permet de montrer que, même mourant, Domitius fait face. Il fait face comme à Corfinium lorsqu'il avait été amené devant son ennemi et déjà auparavant lorsqu'il avait lancé à ses troupes l'ordre de couper le pont enjambant l'Aternus. La composition lucanienne organise ce tout premier face à face dans la structure en chiasme que forment les harangues respectives du chef pompéien et du futur dictateur. À l'expression immergite pontem, qui clôt le vers 484, répond la formule ascendite pontem sur laquelle s'achève le vers 499 . Et tandis que le discours de Domitius commence sur ses ordres donnés à l'impératif ${ }^{48}$ et se poursuit par la description du résultat à obtenir, celui de César commence par la description de l'objectif des ennemis et s'achève sur les ordres à l'impératif ${ }^{49}$. L'effet de symétrie met en valeur l'enjeu du combat: ce pont sur la mention duquel s'ouvre puis se referme l'ensemble constitué par les propos que prononcent les deux chefs. Le premier affrontement entre les deux hommes se déroule donc par discours interposés. Si l'on considère dans leur ensemble les trois paires de discours qui marquent leurs rencontres successives, on constate une évolution à nos yeux significative. Dans le premier cas, les paroles de César répondent à celles de Domitius et sanctionnent sa victoire. Dans le second cas, la défaite de Domitius est outrageusement confirmée par le fait que César est le seul à s'exprimer. Le pompéien, défait, humilié par la grâce que lui accorde l'ennemi, est dans l'incapacité de lui répondre. Cependant, un monologue découvre ses pensées au lecteur, sorte de réplique muette qui annonce un nouvel épisode. Ce ne sera pas Marseille mais Pharsale. Symboliquement, nous assistons alors à un véritable échange dans lequel le vaincu peut se permettre enfin de répondre à celui qui se pense vainqueur. Comme l'a souligné W.D. Lebek, le second dialogue entre les deux hommes constitue le pendant du premier ${ }^{50}$. Cette scène a été rapprochée de deux passages de l'Iliade: la confrontation entre 
Hector et Patrocle mourant, au chant XVI, et le dialogue entre Achille et Hector mourant au chant XXII ${ }^{51}$. Concernant ce rapprochement, deux points ont attiré l'attention des commentateurs. En premier lieu, chez Homère, le dernier mot appartient toujours au vainqueur. En second lieu, le vaincu prononce une prophétie où il annonce la mort de son ennemi ${ }^{52}$. Nous commencerons par le second point. On pourrait penser que Lucain prend le contre-pied du point de vue homérique en faisant exprimer à Domitius un espoir qui s'assimilerait à une prophétie erronée. Voici les dernières paroles du Pompéien dans la traduction de J. Soubiran :

Tu n'as point touché le prix funeste de tes crimes,

tu n'es ni sûr de ton destin ni si grand, César, que ton gendre :

ainsi je te vois moi, libre et serein, toujours aux ordres de Magnus,

en gagnant les ombres du Styx ; toi, dompté par le cruel Mars,

pour Pompée et pour nous tu paieras lourdement :

tel est, mourant, l'espoir qui m'est permis ${ }^{53}$.

Cette traduction a, entre autres, l'immense mérite de conserver l'ambiguïté des derniers mots de Domitius :

te saeuo Marte subactum / Pompeioque grauis poenas nobisque daturum, cum moriar, sperare licet ${ }^{54}$.

On peut en effet les interpréter ainsi : Domitius nourrit l'espoir que César, qui n'a pas encore définitivement triomphé de Pompée, sera vaincu au cours du conflit. Mais il s'agit seulement d'un espoir que la mort toute proche, parce qu'elle l'empêche de voir la suite des événements, lui permet encore de nourrir ${ }^{55}$. Cet espoir, tel qu'il paraît exprimé, ne se réalisera pas. César touchera bel et bien le fruit de ses crimes. Cependant, le destin, quelque peu aidé par Lucain, donne aux propos de Domitius une tonalité prophétique. L'expression saeuo Marte peut aussi renvoyer aux ides de Mars. Dans cette optique, le double datif Pompeio nobisque, qui associe Pompée à Domitius, possède une étrange résonance. En faisant de Magnus le bénéficiaire et non l'acteur de la punition subie par César, il fait écho au futur meurtre du dictateur. N'oublions pas l'anecdote rapportée par Plutarque, selon laquelle César, percé de coups, se serait effondré au pied de la statue de Pompée ${ }^{56}$. En outre, ni César ni Domitius ne se sont aperçus que, sur le champ de bataille, se trouvait justement Brutus, incognito, déjà prêt à frapper le tyran mais qui devra attendre son triomphe pour que le destin l'autorise à accomplir son geste ${ }^{57}$. Ainsi, sans le savoir, Domitius se fait la voix du destin. Quant à César, il est pris au piège de ses propres sarcasmes. Des commentateurs ont souligné, à juste titre, le caractère amphibologique de l'apostrophe qu'il lance à son ennemi lorsqu'il l'aperçoit au milieu des mourants: successor Domiti ${ }^{58}$. Le terme successor évoque, avec une ironie mordante, le fait que Domitius avait été désigné par le Sénat pour lui succéder à la tête de la Gaule cisalpine. Cependant, un lecteur contemporain de Lucain ne pouvait s'empêcher de songer à Néron, descendant de Domitius et devenu, d'une certaine manière, successeur de César. Le destin prend donc les deux hommes au mot, mais au détriment du futur dictateur.

Il nous semble ainsi que, si la réplique de Domitius clôt le court dialogue entre les deux hommes, c'est bien, à certains égards, dans la ligne du topos homérique, et donc parce que le Pompéien remporte une forme de victoire sur son adversaire. Lucain écrit à ce propos: Salua libertate perit ${ }^{59}$. On comprend évidemment que l'acceptation du pardon césarien impliquait, pour le défenseur de la République, un asservissement au tyran. Accepter de rester en vie, c'était se soumettre ${ }^{60}$. Par conséquent, seule la mort permet de marquer le refus de la soumission et l'affirmation conjointe de la liberté. Domitius annonce, par son attitude à Pharsale, le suicide de Caton refusant le pardon de César ${ }^{61}$. 
Mais il faut souligner, dans le cas de Domitius, la valeur rédemptrice du trépas, qui lui permet de restaurer une intégrité morale que sa survie à la défaite de Corfinium avait entamée. Alors que sa nobilitas exigeait le supplice, le pardon césarien l'en avait privé. Significativement, le poète signale la joie de Domitius à l'idée qu'en mourant, il échappe à un second pardon. C'est qu'en fait, sa liberté ne pouvait être sauvegardée qu'à la condition d'effacer la compromission née de la grâce qui lui avait été accordée. Cela nécessitait d'être tué en combattant celui-là même qui lui avait laissé la vie.

On peut aller plus loin. Un élément, dans les propos que César adresse au mourant, doit nous alerter :

Iam Magni deseris arma / successor Domiti ; sine te iam bella geruntur ${ }^{62}$.

L'expression Magni deseris arma n'est pas sans rappeler la version de la mort de Domitius qui est exposée dans le Bellum Ciuile. En mourant, en effet, Domitius déserte le champ de bataille ${ }^{63}$. Qui est ce Domiti auquel s'adresse César? Non le pugnax évoqué par Lucain, mais le successor décrit comme un lâche par le mémorialiste. Il n'est sans doute pas indifférent que l'interjection césarienne prenne ici la place de l'apostrophe lucanienne ${ }^{64}$. Derrière le protagoniste du récit, c'est bien le futur auteur du Bellum Ciuile qui transparaît, tentant d'enfermer Domitius dans la persona de l'homme lâche qu'il lui conférera dans toute son œuvre. Ce processus avait été entamé lors de la première confrontation entre les deux hommes, quand le vainqueur avait refusé d'accéder à la demande de son prisonnier qui réclamait le supplice :

Viue licet nolis et nostro munere, dixit, cerne diem. Victis iam spes bona partibus esto exemplumque mei ${ }^{65}$.

L'expression esto exemplum mei est très claire : Domitius se trouve transformé malgré lui en instrument de la propagande de César. Il participe contre son gré à cette image de douceur (lenitas) que ce dernier entend donner de lui-même. À l'inverse, l'image de Domitius subit une profonde dégradation. De cela, le pompéien est parfaitement conscient, comme l'indiquent, dans son monologue, les vers 522-523 ${ }^{66}$ :

Romamne petes pacisque recessus / degener?

19 Lui à qui la mort a échappé, devient celui qui a échappé à la mort: terrible renversement des termes où se fonde l'image d'un homme en fuite. Contre l'aliénation engendrée par le pardon de César, la seule solution consiste à se lancer à corps perdu dans la quête d'une mort héroïque, les armes à la main, celle que mentionne Sénèque dans l'évocation du suicide manqué : rogo mortem armatus ${ }^{67}$. C'est bien ce qui ressort de la réflexion à laquelle se livre le personnage décrit par Lucain :

rue certus et omnes / lucis rumpe moras et Caesaris effuge munus ${ }^{68}$.

On retrouve ici le thème de la fuite, mais détourné de son sens originel : ce n'est pas la mort que veut fuir Domitius mais le piège du munus dans lequel il s'est laissé enfermer. Au suicide manqué décrit dans les autres textes Lucain substitue ce trépas héroïque que César lui dérobe et qu'il lui faut accomplir pour reconquérir à la fois sa liberté et son identité véritable. On comprend, dès lors, l'effacement de son action à Marseille, nécessaire au tracé d'une trajectoire tout entière tendue vers une mort salvatrice.

En ce sens, le face à face de Pharsale marque la victoire paradoxale de Domitius, dont la joie de mourir est soulignée par le poète :

laetus / labitur ac uenia gaudet caruisse secunda ${ }^{69}$.

Malgré les tentatives de César pour subvertir sa natura véritable, c'est-à-dire la natura que lui confère Lucain, le Pompéien peut se dire "libre» (liber) et «serein» (securus) car il meurt au service de Magnus ${ }^{70}$, proclamant ainsi à la fois sa uirtus et sa 
fides. C'est peut-être ce qui, au seuil de la mort, justifie en lui ce sentiment de securitas : la certitude d'être en plein accord avec soi-même, d'affirmer, en dépit de César, sa véritable identité.

Le traitement de la figure de Lucius Domitius Ahenobarbus chez Lucain doit donc être considéré globalement, dans la trajectoire qui le conduit de Corfinium à Pharsale. S'il est clair que le poète remodèle le personnage selon ses propres conceptions, qu'il crée des situations symboliques s'écartant de l'Histoire, il faut se garder de penser que sa vision ne serait que personnelle, et totalement artificieuse. Lucain se réfère sans doute en partie à une tradition historiographique hostile à César. Et l'on ne peut prendre pour modèle de vérité l'image de Domitius que ce dernier trace dans son Bellum ciuile. C'est d'ailleurs là que se trouve peut-être l'un des intérêts majeurs du portrait lucanien. Nous avons vu que le poète prenait le contre-pied de César. Renversant la perspective que celui-ci conférait à l'itinéraire historique du Pompéien, il transforme en quête obstinée de la mort ce qui était, dans le récit césarien, une fuite perpétuelle. Mais surtout, jouant sur la référence intertextuelle, il met en scène l'opposition d'un Domitius idéalisé au personnage que César a tenté de lui faire jouer. Figure-symbole, Domitius n'incarne pas seulement la résistance à une entreprise politique de destruction de la respublica, il incarne aussi la volonté lucanienne de dénoncer une réécriture du passé qui affecte l'image de ceux qui en furent les acteurs. Dans cette optique, nous nous situerons donc du côté de ceux qui considèrent que le portrait laudatif du trisaïeul de Néron n'est pas à mettre en rapport direct avec les liens qui ont pu exister entre Lucain et l'empereur ${ }^{71}$. Autrement dit, nous ne croyons pas que Lucain ait glorifié Domitius dans le but de flatter Néron. La charge symbolique et la cohérence du personnage rendent cette hypothèse difficile à admettre. Cela n'exclut pas que le poète ait pu songer, un temps, qu'une telle parenté pourrait rendre sa vision du héros de la République plus acceptable. Mais ces considérations ont été vite balayées par la dégradation de ses relations avec le prince. Il était temps pour Lucain de passer de l'autre côté du miroir, de devenir à son tour un " personnage », soumis aux partis pris des historiographes.

\section{BIBLIOGRAPHIE}

\section{Textes anciens}

Cicéron, Correspondance, éd. J. Bayet, CUF, Paris, 2002 [1964].

Lucain, La Guerre civile : VI 333 - X 546, éd. J. Soubiran, Monde antique, Toulouse, 1998.

Lucan, Civil war VIII, éd. R. Mayer, Warminster, 1981. 


\section{Textes modernes}

AHL F.M. 1971, « Lucan's De Incendio Vrbis, Epistulae ex Campania and Nero's ban », TAPhA 102, p. 1-27.

BRISSET J. 1964, Les idées politiques de Lucain, Coll. d'études anciennes, Paris.

BURNS A. 1966, « Pompey's Strategy and Domitius' Stand at Corfinium », Historia 15, p. 74-95.

ColLINS J.H. 1972, « Caesar as Political Propagandist », ANRW I, 1, p. 922-966.

FRITZ K. von 1942, « Pompey's Policy before and after the Outbreak of the Civil War of 49 B.C. », TAPhA 73, p. 145-180.

GRIMAL P. 1970, « Le poète et l'histoire », in Lucain : sept exposés suivis de discussions, Vandœuvres Genève du 26 au 31 août 1968, Entretiens sur l'Antiquité classique 15, Genève, p. 51-117.

KARDOS M.-J. 2006, « La “déformation historique” dans le livre I du Bellum ciuile : le témoignage de Cicéron et de la correspondance », VL 175, p. 19-35.

LEBEK W.D. 1976, Lucans Pharsalia: Dichtungsstruktur und Zeitbezug, Hypomnemata 44, Göttingen. LE BOHEC Y. 2001, César chef de guerre : César stratège et tacticien, L’art de la guerre, Monaco.

LEIGH M. 1997, Lucan: Spectacle and Engagement, Oxford Classical Monographs, Oxford.

LINTOTT A.W. 1971, « Lucan and the History of the Civil War », CQ 21, p. 488-505.

LOUNSBURY R. 1975, « The Death of Domitius in the Pharsalia », TAPhA 105, p. 209-212.

MAKOWSKI J.F. 1974, Death and Liberty in Lucan's Pharsalia [Thesis (Ph. D.), Princeton University]. MARTIN P.M. 2000, La Guerre des Gaules, La Guerre civile, César, Textes fondateurs, Paris.

MASTERS J. 1994, « Deceiving the Reader: the Political Mission of Lucan's Bellum Civile », in J. Elsner, J. Masters (éds), Reflections of Nero: Culture, History, and Representation, Londres, p. 151-177.

PICHON R. 1912, Les sources de Lucain, Paris.

RAMBAUD M. 1953, L'art de la déformation historique dans les Commentaires de César, Annales de l'université de Lyon. Lettres 23, Paris.

RAMBAUD M. 1960, « L'opposition de Lucain au Bellum Ciuile de César », Information littéraire 4, p. $155-162$.

VEITH G. 1913, « Corfinium. Eine kriegsgeschichtliche Studie », Klio 13, p. 1-26.

ZECCHINI G. 2010, « Prospettive di storia romana sotto Nerone: Lucano tra i due Domizî », in O. Devillers, S. Franchet d'Espèrey (éds), Lucain en débat : rhétorique, poétique et histoire : actes du colloque international, Institut Ausonius, Pessac, 12-14 juin 2008, Études 29, Pessac - Paris, p. 295-302.

\section{NOTES}

1. Dans la discussion qui suit la communication de Grimal 1970, p. 108. Lire aussi Lintott 1971, p. 489.

2. Pour éviter tout risque de confusion avec le Bellum ciuile de César, nous utiliserons le titre Pharsale pour désigner l'œuvre de Lucain.

3. Rambaud 1953, p. 13-14. 
4. César, B.C. 2, 31, 1. Lire Le Bohec 2001, p. 326 sq. L'événement a été, en tant que tel, l'objet de la longue étude de Veith 1913.

5. Alterum existimari conseruatorem inimicorum, alterum desertorem amicorum, «L'un a la réputation de sauver ses ennemis l'autre de trahir ses amis ", écrit Cicéron à Atticus le 25 février (Cicéron, Att. 8, 9b, 1). La tentative de résistance de Domitius semble avoir suscité de grands espoirs dans le camp républicain, d'où la rancœur provoquée par l'attitude de Pompée. Kardos 2006, p. 26-27.

6. Luc., 2, 392-609.

7. Nescius interea capti ducis arma parabat / Magnus, «Cependant Magnus, ignorant la capture du général, préparait ses armes» (Luc., 2, 526-527). Rambaud 1960, p. 158 et 162; Martin 2000, p. 128.

8. Pompée semble n'avoir pas clairement dévoilé ses desseins. Domitius n'aurait pas su qu'il souhaitait évacuer l'Italie. Lire Fritz 1942, p. 154 sq. ; Burns 1966, p. 83 sq.

9. En Att. VIII, 1, 3, Cicéron signale la sottise du personnage. Cependant Fritz 1942, p. 163 sq., démontre qu'en partant du principe que Domitius ignorait bel et bien les plans de Pompée, sa tentative pour « fixer " César à Corfinium peut tout à fait se justifier. Lire également Burns 1966, p. 84. Contra: Rambaud 1960, p. 161-162; Martin 2000, p. 128.

10. Pompeius, his rebus cognitis quae erant ad Corfinium gestae, Luceria proficiscitur Canusium atque inde Brundusium (Caes., B.C. 1, 24, 1).

11. La capitulation de Corfinium a lieu le 21 février alors que Pompée quitte Lucérie le 18 ou le 19 (Cic., Att. 8, 12a).

12. Cum uultus Domiti cum oratione non consentiret, atque omnia trepidantius timidiusque ageret, quam superioribus diebus consuesset, multumque cum suis consiliandi causa secreto praeter consuetudinem colloqueretur, concilia conventusque hominum fugeret, res diutius tegi dissimularique non potuit (Caes., B.C. 1, 19).

13. Reseratis [...] portis / captiuum traxere ducem, ciuisque superbi / constitit ante pedes (Luc., 2, 507-509).

14. "Quant à Domitius, on dit que, à cette nouvelle, il s'est rendu, ainsi que ceux qui étaient avec lui » (Cic, Att. 8, 8, 2). Par la suite, Cicéron cherchera plusieurs fois à prendre de ses nouvelles : Kardos 2006, p. 28 et p. 34, n. 67.

15. «Une fois répandue la nouvelle de la décision de Domitius » (Caes., B.C. 1, 20, 1).

16. Lentulus Spinther, Vibullius Rufus, Lucilius Hirrus, par ex. Lire Collins 1972, p. 949 sq.

17. Pluris e familia cognosci referre arbitror, quo facilius appareat ita degenerasse a suorum uirtutibus Nero, ut tamen uitia cuiusque quasi tradita et ingentia rettulerit, "Je crois qu'il importe de faire connaître plusieurs membres de cette famille afin de pouvoir mieux montrer que si Néron dégénéra des vertus de ses ancêtres, inversement les vices de chacun d'eux se retrouvèrent en lui comme s'ils avaient été transmis par le sang » (Suet, Nero 1,6).

18. Quant à Appien, il indique, en VI, 38, que Domitius aurait été capturé par les habitants, aux portes de la ville, alors qu'il cherchait à s'enfuir. Mais il pourrait s'agir d'une extrapolation fondée sur le récit de César. On ne peut s'empêcher de songer à l'épisode de la capitulation de Sulmone, qui a lieu au même moment. Les habitants ouvrent les portes de la ville à Antoine, tandis que Lucretius et Attius, qui commandaient la garnison, tentent de s'échapper en sautant au bas des remparts. Attius est pris et amené devant Antoine (Caes., B.C. 1, 18, 1-3). Sur le manque de fiabilité du récit d'Appien, lire Burns 1966, p. 89.

19. Il commandait manifestement une aile de l'armée pompéienne : à l'exception de César qui, sciemment, ne le mentionne pas à ce poste, les auteurs anciens le placent soit à la tête de l'aile droite, soit à celle de l'aile gauche. Lire Pichon 1912, p. 115-116.

20. Lire Fritz 1942, p. 165 ; Burns 1966, p. 86 sq., qui évoque également, p. 87, l'hypothèse selon laquelle Domitius a pu prononcer son discours d'encouragement à combattre avant de recevoir la lettre de Pompée et non après sa lecture.

21. D.C., 41, 10-11. 
22. Le récit de César pose d'autres difficultés. Si les hommes se rassemblent le 20 février au soir pour prendre leur décision, alors que la lettre de Pompée, écrite le 17, n'est arrivée au mieux que deux jours plus tard, il faudrait que le prétendu changement d'attitude de Domitius ait été perceptible en un laps de temps très court. On peut objecter que Pompée avait déjà refusé son aide dans une lettre datant du 16 février (Cic., Att. 8, 12c), mais César, justement, n'en dit mot. Pour la chronologie de l'échange épistolaire, nous nous référons à J. Bayet (éd.), Cicéron. Correspondance, Paris, 2002 [1964], p. 139 sq. L'évocation du revirement du contingent marse, qui refuse d'abord de rejoindre les mutins avant de se rallier à eux, pourrait être une habile reconstruction des dissensions qui eurent lieu au moment de lâcher l'état-major pompéien : leur ignorance du projet de Domitius, invoquée par César pour justifier leur désaccord initial, paraît contradictoire avec l'affirmation selon laquelle la nouvelle de la trahison du chef s'était répandue dans la troupe. Par ailleurs, il signale que les Marses, sitôt après leur refus, vont occuper l'endroit le mieux fortifié de la place. Ce point pourrait, en réalité, être mis en relation avec le rejet d'un projet de sortie. Autre élément troublant: plus tard, en Afrique, c'est Curion qui doit empêcher ses hommes de rejoindre le Pompéien Quintilius Varus. Or, ce dernier faisait partie des compagnons de Domitius conspués par les légions révoltées au moment où elles livraient leur état-major à César (B.C. 1, 23, 2) et ce sont ces mêmes légions qui composent les troupes tentées cette fois de repasser du côté des Pompéiens : deux centurions marses justement, suivis de vingtdeux hommes, ont déjà fui le camp césarien, lorsque Curion s'adresse à l'armée. On constate donc, à tout le moins, qu'en temps de guerre civile, on passe facilement d'un parti à l'autre. César lui-même est contraint de rappeler, à cette occasion, que l'on traite de transfuges les hommes de Curion, même si ce dernier, conformément à la version du Bellum Ciuile, accuse à nouveau Domitius de trahison (Caes., B.C. 2,$28 ; 32,8$ ).

23. Burns 1966, p. 89, n. 85.

24. Zecchini 2010.

25. Mortem rogo armatus, « je demande la mort, les armes à la main » (Sen., De ben. 3, 24).

26. Suet., Nero 2, 4.

27. Plu., Caes. 34, 6-8.

28. Adeo esse perterritos nonnullos ut suae uitae durius consulere cogantur (Caes., B.C. 1, 22, 6).

29. L'intervention de Domitius aurait eu lieu dès le début des hostilités, selon César, B.C. 1, 34, 1-3, qui souligne ainsi l'hypocrisie supposée des Marseillais. Mais Suétone laisse entendre, dans Nero 2, 4, que l'ancêtre de Néron aurait apporté son soutien à la cité après le début du siège. Quoi qu'il en soit, Domitius, en tant que proconsul, était officiellement le seul représentant de Rome dans la région. Par ailleurs, la manière dont il constitue sa flotte et sa tentative réussie pour fuir la ville sur le point de capituler offrent au mémorialiste autant de prétextes pour le dénigrer (Caes., B.C. $1,34,1-2 ; 2,22,2-4)$. Il faut ajouter que César insiste sur l'impiété des défenseurs de la cité, qui attaquent les assiégeants pendant une trêve (Caes., B.C. 2, 12-14).

30. Luc., 3, 298-762.

31. «[Domitius] que les destins menaient parmi tous les désastres : pas un endroit où la Fortune de Magnus ait succombé sans lui » (Luc., 7, 600-601).

32. «Tant de fois vaincu par César » (Luc., 7, 602).

33. Luc., 2, 522-525 ; 7, 219-220. Nous rejoignons l'opinion de Brisset 1964, p. 35.

34. «Lucius Domitius, qui avait quitté le camp pour se réfugier dans la montagne, à bout de forces, fut tué par les cavaliers » (Caes., B.C. 3, 99, 5).

35. On ne peut que constater le manque de clarté de la formule employée par Cicéron dans Phil. 2, 71: L. Domitium clarissimum et nobilissimum uirum occideras multosque praeterea, qui e proelio effugerant, quos Caesar ut nonullos fortasse seruauisset crudelissime persecutus trucidaras, «Tu avais tué Lucius Domitius, très illustre et très noble citoyen, et en outre, tu avais poursuivi et massacré avec la plus grande cruauté beaucoup d'autres, qui avaient échappé au combat et que César, 
comme il l'a fait pour quelques-uns, aurait peut-être épargnés ». Sur ce point, lire Masters 1994, p. 174, n. 54 .

36. Nous renvoyons aux intéressantes remarques de Lounsbury 1975.

37. Luc., 7, 599-616.

38. Citons Pichon 1912, p. 156 : «même en ce qui concerne Domitius, c'est-à-dire sur un point où tout se réunissait pour engager le poète à modifier la réalité historique, il ne paraît pas l'avoir altérée plus que Tite-Live lui-même n'avait dû le faire ». Lire également Brisset 1964, p. 134-135 ; Masters 1994, p. 151.

39. Lire Rambaud 1953, p. 347-348.

40. Martin 2000, p. 126.

41. Rambaud 1960, p. 155 sq.

42. Masters 1994, p. 165 sq.

43. Suétone met l'accent sur l'inconstance d'un personnage qui se serait pourtant voulu inflexible : il le définit comme neque satis constans et ingenio truci, «à la fois manquant de fermeté et d'une nature farouche ». Il rapporte qu'au moment où Pompée se demandait comment il fallait traiter ceux qui, dans le conflit, souhaitaient rester neutres, il fut le seul, dans l'entourage de Magnus, à réclamer qu'ils soient considérés comme des ennemis (Suet. Nero 2, 4-5).

44. Luc., 2,$479 ; 7,219 ; 7,600$.

45. Vultu tamen alta minaci / nobilitas recta ferrum ceruice poposcit, « c'est la tête droite et le regard menaçant que cette âme d'une haute noblesse réclame le fer » (Luc., 2, 509-510).

46. «Une mort pourtant se fit remarquer dans ce carnage de grands hommes, celle de l'opiniâtre Domitius » (Luc., 7, 599-600).

47. Luc. 7, 603-604.

48. "Socii, decurrite», dixit, / "fluminis ad ripas undaeque immergite pontem», "Camarades, dit-il, courez jusqu'aux rives du fleuve et submergez le pont » (Luc., 2, 483-484).

49. Equitum properate cateruae, / ite simul pedites, ruiturum ascentidte pontem, "hâtez-vous escadrons, fantassins, accompagnez-les, montez sur le pont prêt de s'écrouler » (Luc., 2, 498-499). Sur l'ensemble du passage, lire l'analyse de Lebek 1976, p. 147 sq.

50. Lebek 1976, p. 264.

51. Hom., Il. 16, 818-861 ; 22, 247-366.

52. Lebeck 1976, p. 264 sq. ; Masters 1994, p. 164 sq.

53. Non te funesta scelerum mercede potitum / sed dubium fati, Caesar, generoque minorem / aspiciens Stygias Magno duce liber ad umbras / et securus eo: te, saeuo Marte subactum, / Pompeioque grauis poenas nobisque daturum, / cum moriar, sperare licet (Luc., 7, 610-614), Soubiran (éd.) 1998, p. 75.

54. Luc., 7, 613-614.

55. Sur l'expression cum moriar, lire la remarque de Masters 1994, p. 165.

56. Plu., Caes. 66, 12-13.

57. Viuat et, ut Bruti procumbat uictima, regnet !, « Qu'il vive et pour tomber victime de Brutus, qu'il règne!» (Luc., 7, 596).

58. Par ex., Masters 1994, p. 174, n. 51.

59. «Il périt en ayant sauvegardé sa liberté » (Luc. 7, 602-603).

60. Sur ce point, lire en particulier l'analyse de Makowski 1974, p. 9 sq. Sur la clementia de César telle qu'elle apparaît chez Lucain, lire Leigh 1997, p. 53-68.

61. Sur la mort de Domitius conçue comme une deuotio, lire Leigh 1997, p. 141-143. Par ailleurs, on ne soulignera jamais assez l'importance que revêt, chez les Anciens, la possibilité, par la mort, d'échapper à la contemplation des malheurs qui vont s'abattre sur la communauté (par ex. Tacite, Ag. 44, 6-45, 1 ; Ann. 6, 48, 1-3). Domitius exprime donc avec force sa joie de mourir sans connaître le joug imposé par César.

62. «Désormais tu abandonnes les armes de Magnus, Domitius mon successeur; sans toi désormais la guerre se poursuit» (Luc., 7, 606-607). 
63. Lire Masters 1994, p. 167.

64. Luc., 2, 479.

65. «Vis malgré toi, dit-il, et vois le jour par notre bienfait. Sois désormais pour le parti vaincu un gage d'espoir et un exemple de ce que je suis » (Luc., 2, 512-514).

66. « Gagneras-tu Rome et de paisibles retraites en dégénéré ?» (Luc., 2, 522-523).

67. Lire supra, n. 25.

68. «Cours sans hésiter, romps tous les délais de la vie et fuis le présent de César » (Luc., 2, 524-525).

69. «Joyeux, il s'effondre et se réjouit d'éviter un deuxième pardon » (Luc., 7, 603-604).

70. Luc., 7, 612-613.

71. Ahl 1971, p. 13, et p.19-24 ; Lownsburry 1975, p. 209 ; Soubiran (éd.) 1998, p. 194. Contra : Brisset 1964, p. 188-189; Mayer (éd.) 1981, p. 7.

\section{RÉSUMÉS}

En réponse à l'idée que le compte rendu des actes du chef pompéien Domitius Ahenobarbus chez Lucain, considéré comme déviant par rapport à la réalité historique, détermine une conception du personnage tout aussi éloignée de la véritable nature de l'individu, l'article explique pourquoi analyser la manière dont le poète représente le comportement de Domitius implique de s'interroger sur la validité de son traitement historiographique. Cette étape préliminaire permet ensuite d'éclairer la caractérisation du personnage en fonction de la double confrontation dialectique qui le met aux prises avec César. Figure-symbole, Domitius n'incarne pas seulement la résistance à une entreprise politique de destruction de la respublica, il incarne aussi la volonté lucanienne de dénoncer une réécriture du passé qui affecte l'image de ceux qui en furent les acteurs.

\section{INDEX}

Mots-clés : clementia, déformation historique, épopée, épopée historique romaine, guerre civile romaine, histoire et idéologie, historiographie antique, historiographie romaine, intertextualité, lenitas, libertas, mort héroïque, narration, prosopographie impériale romaine, valeurs épiques, vérité historique

Keywords : clementia, historical distorsions, epic, Roman historical epic, Roman civil war, history and ideology, ancient historiography, Roman historiography, intertextuality, lenitas, libertas, heroic death, narration, Roman imperial prosopography, ethic values in epic, historical truth

nomsmotscles Appien, César, Cicéron, Dion Cassius, Homère, Lucain, Plutarque, Sénèque, Suétone

Index géographique : Apennins, Aternus, Brindes, Corfinium, Gaule, Lucérie, Marseille, Pharsale, Picenum 